\title{
Potencial reprodutivo (PR) de touros Nelore avaliados por parâmetros andrológicos e comportamento sexual. Classificação por pontos: nova proposição
}

\author{
[Reproductive potential of Nelore bulls evaluated by andrological parameters \\ and sexual behavior. Classification by points - a new proposition] \\ V.O. Fonseca ${ }^{1}$, C.S. Franco ${ }^{2}$, N.A. Azevedo ${ }^{3}$, L.Z. Oliveira ${ }^{1 *}$, \\ G.A. Monteiro ${ }^{1}$, L.F.L. Cavalcanti ${ }^{1}$, L.R. Molina ${ }^{1}$ \\ ${ }^{1}$ Escola de Veterinária - Universidade Federal de Minas Gerais - Belo Horizonte, MG \\ ${ }^{2}$ Fazenda Jatobá - Brasilândia, MS \\ ${ }^{3}$ Empresa de Pesquisa Agropecuária de Minas Gerais - Belo Horizonte, MG
}

\begin{abstract}
RESUMO
Este estudo teve por objetivo comparar variações de parâmetros andrológicos e comportamentais de touros Nelore de diferentes faixas etárias, calcular seu potencial reprodutivo (PR) e propor uma nova tabela de classificação por pontos, de acordo com as médias atualmente alcançadas por eles nas características estudadas. Foram utilizados dados de 6162 exames andrológicos de touros da raça Nelore, entre 12 e 80 meses de idade, em regime de monta natural. O número de touros classificados como aptos consistiu em $88,9 \%$ dos animais avaliados $(\mathrm{n}=5480)$, sendo $51,6 \%$ desses considerados excelentes $(\mathrm{n}=2827), 41,2 \%$ muito bons $(\mathrm{n}=2257)$ e $7,2 \%$ considerados bons $(\mathrm{n}=394)$. Entre os animais questionáveis $(\mathrm{n}=682 ; 11,1 \%), 79,6 \%$ foram classificados como inaptos temporários $(\mathrm{n}=542)$ e $20,4 \%(\mathrm{n}=139)$ como animais descarte, de acordo com o exame andrológico, independentemente do teste da libido. O número de touros classificados como excelentes se reduziu para 752 (12,2\%) quando dados de comportamento sexual foram incluídos para definição do seu PR. Concluiu-se que o uso de tabelas de classificação andrológica por pontos com atualizações técnicas beneficia a seleção mais apurada de touros Nelore. $\mathrm{O}$ teste da libido é ferramenta importante para a determinação do PR, o qual permite melhor aproveitamento dos reprodutores.
\end{abstract}

Palavras-chave: andrologia, bovino, libido, perímetro escrotal, potencial reprodutivo

\begin{abstract}
This study aimed to compare variations of andrological and behavioral parameters from Nelore bulls of different ages, to calculate their reproductive potential $(R P)$ and propose a new classification table by points, considering current averages in each reproductive trait studied. Data were collected from 6162 breeding soundness examinations of Nelore bulls aged between 12 and 80 months, under natural mating. According to andrological parameters, regardless of the libido test, the number of bulls classified as approved was $88.9 \%(n=5480)$, being $51.6 \%$ considered as excellent $(n=2827), 41.2 \%$ very good $(n=$ $2257)$ and $7.2 \%$ considered as good $(n=394)$. Among the animals considered as questionable $(n=682$; $11.1 \%), 79.6 \%$ were classified as temporarily reproved $(n=542)$ and $20.4 \%(n=139)$ as discarded animals. The number of bulls classified as excellent decreased to $752(12.2 \%)$ when sexual behavior data were included to define their RP. It was concluded that the use of tables for andrological classification by points with technical updates improves the reproductive selection of Nelore bulls. The libido test is an important tool for RP determination which provides better utilization of the sires.
\end{abstract}

Keywords: andrology, bovine, libido, scrotal perimeter, reproductive potential

Recebido em 24 de setembro de 2018

Aceito em 22 de março de 2019

*Autor para correspondência (corresponding author)

E-mail: leticiazoccolaro@yahoo.com.br 


\section{INTRODUÇÃO}

O Brasil tem no agronegócio uma de suas principais atividades econômicas, alicerçando, inclusive, setores secundários e terciários da economia nacional. Quando bem assessorada e estruturada essa atividade, o seu custo/benefício é extremamente positivo. Considerando que a melhoria na eficiência reprodutiva pode impactar positivamente a lucratividade do rebanho, destaca-se a relevância da utilização de reprodutores com alto potencial para fertilidade na produtividade de uma criação bovina (Fonseca et al., 2019).

A seleção de touros de elevada eficiência reprodutiva apresenta grande importância econômica. A consequência dessa seleção é a possibilidade de incluir um maior número de vacas por touro ao que tem sido rotineiramente empregado na pecuária brasileira, isto é, a razão de 25:1 (Fonseca et al., 1997b; 2000; Franco et al., 2006). Esse baixo desafio aplicado ao reprodutor dificulta o melhoramento do rebanho, pois acarreta a utilização de touros de baixa capacidade reprodutiva e genética, aumentado os custos de produção.

A partir da década de 90, começaram a aparecer, no Brasil, várias tabelas de classificação de touros por pontos, de forma a selecionar aqueles com maior capacidade reprodutiva (Fonseca et al., 1989, 1997ª 2000; Vale-Filho, 2001; ValeFilho et al., 2010; Fonseca et al., 2019). Essas classificações seguem os fundamentos da Sociedade Americana de Theriogenologia (Chenoweth, 1984), os quais, porém, foram adaptados para touros zebuínos (Bos indicus), de acordo com particularidades de biometria testicular e aspectos físicos e morfológicos do sêmen. Essas características possuem forte componente genético, sendo algumas delas (como idade à puberdade e perímetro escrotal) de herdabilidade média a alta (Bergmann et al., 1996; Pereira et al., 2002). Nesse sentido, destaca-se o perímetro escrotal (PE), medida de prática aferição que, além de alta repetibilidade e herdabilidade, apresenta forte correlação positiva com a capacidade reprodutiva e a produção seminal (Bergman et al., 1996; Ahmad et al., 2010; Ayala et al., 2016). Também, aspectos físicos e morfológicos do sêmen apresentam repetibilidade de média a alta, além de correlações elevadas com diversos parâmetros reprodutivos (Fonseca, 2009; Fonseca et al., 2019) e potencial para herdabilidade (Pereira, 2012).

Embora a libido seja de herdabilidade pouco conhecida, ela apresenta alta correlação com a capacidade de serviço, cuja herdabilidade apresenta-se alta $(\mathrm{h}=0,59 ;$ Blockey, 1978). Destaca-se, portanto, que o comportamento sexual oferece importante complementação ao exame andrológico propriamente dito, uma vez que tem papel fundamental na correta determinação do potencial reprodutivo (PR) do macho bovino (Fonseca et al., 1991). Diante do exposto, os objetivos deste trabalho foram estudar as variações dos parâmetros andrológicos e do comportamento sexual de touros da raça Nelore de diferentes faixas etárias e em atividade no campo, bem como propor uma nova tabela de classificação por pontos (tendo-se em vista as médias atualmente alcançadas por eles em cada característica estudada).

\section{MATERIAL E MÉTODOS}

Para o presente estudo, foram utilizados dados de 6162 exames andrológicos de touros da raça Nelore (Bos taurus indicus), criados a pasto, em regime de monta natural. Os animais avaliados apresentavam faixa etária de 12 até 80 meses, oriundos de 21 fazendas de seis estados brasileiros (São Paulo, Minas Gerais, Mato Grosso, Mato Grosso do Sul, Goiás, Bahia). Estabeleceram-se, entre eles, cinco faixas etárias: de 12 a 18 , de 18 a 24, de 24 a 36, de 36 a 48 e acima de 48 meses.

O exame andrológico, denominado "teste 1", consistiu em exame clínico geral e reprodutivo, aferição do perímetro escrotal (PE) e avaliação dos aspectos físicos (motilidade $=\mathrm{Mot}$, vigor $=\mathrm{V}$ e turbilhonamento=Tb) e morfológicos do sêmen (defeitos maiores=DM, defeitos menores $=\mathrm{dm}$ e total de defeitos espermáticos=TDE; Blom, 1973), de acordo com o Colégio Brasileiro de Reprodução Animal (Manual..., 1998). A análise da biometria testicular se limitou à aferição do perímetro escrotal $(\mathrm{PE})$ devido à alta correlação $(\mathrm{r}=0,94)$ existente entre $\mathrm{PE}$ e os aspectos biométricos determinantes do volume final dos testículos (Willet e Ohms, 1975). Para a obtenção do sêmen, utilizou-se o método da eletroejaculação $\quad\left(\right.$ Eletrojet $^{\circledR}$, Eletrovet, São Paulo, SP, Brasil), e a avaliação seminal foi 
realizada conforme descrito por Fonseca et al. (2019).

Mediante os valores atribuídos em cada análise, foi possível determinar a classificação andrológica em quatro classes: excelente, muito bom,bom e questionável, com base na tabela específica para touros Bos indicus (Tab. 1; Fonseca et al., 1997a). Foram considerados questionáveis os animais que não atingiram os índices mínimos em pelo menos uma das características estudadas. Esses animais foram divididos em dois grupos: inaptos temporários (Q1) e descarte (Q2). Os animais foram considerados Q1 quando apresentavam uma condição indesejada adquirida e recuperável, enquanto, nos animais descarte (Q2), a causa de sua recusa foi considerada insanável e/ou de origem genética.

Tabela 1. Classificação andrológica de touros Nelore (Bos indicus), com base na circunferência escrotal e em características físico-morfológicas do sêmen, proposta por Fonseca et al. (1997a)

\begin{tabular}{|c|c|c|c|c|}
\hline \multirow{2}{*}{ Parâmetros } & \multicolumn{4}{|c|}{ Classificação } \\
\hline & Excelente & Muito bom & Bom & Questionável \\
\hline \multicolumn{5}{|l|}{ 1-Motilidade espermática } \\
\hline Vigor & 5 & $4<5$ & $3<4$ & $<3$ \\
\hline Motilidade progressiva (\%) & $\geq 75$ & $60<75$ & $30<60$ & $<30$ \\
\hline Pontos outorgados & $21<25$ & $16<21$ & $10<16$ & $<10$ \\
\hline \multicolumn{5}{|l|}{ 2-Morfologia espermática } \\
\hline Defeitos maiores $(\%)$ & $\leq 5$ & $>5-10$ & $>10-20$ & $>20$ \\
\hline Defeitos totais $(\%)$ & $\leq 10$ & $>10-15$ & $>15-30$ & $>30$ \\
\hline Pontos outorgados & $30<35$ & $25<30$ & $15<25$ & $<15$ \\
\hline \multicolumn{5}{|l|}{ 3-Perímetro escrotal $(\mathrm{cm})$} \\
\hline \multicolumn{5}{|l|}{ Idade em meses } \\
\hline $07 a<12$ & $\geq 21,0$ & $19,5<21,0$ & $17,5<19,5$ & $<17,5$ \\
\hline $12 a<18$ & $\geq 26,0$ & $24,0<26,0$ & $21,5<24,0$ & $<21,5$ \\
\hline $18 a<24$ & $\geq 31,5$ & $28,5<31,5$ & $26,0<28,5$ & $<26,0$ \\
\hline $24 a<36$ & $\geq 35,0$ & $32,0<35,0$ & $29,0<32,0$ & $<29,0$ \\
\hline $36 \mathrm{a}<48$ & $\geq 37,0$ & $33,5<37,0$ & $30,5<33,5$ & $<30,5$ \\
\hline$\geq 48$ & $\geq 39,0$ & $36,0<39,0$ & $33,0<36,0$ & $<33,0$ \\
\hline Pontos outorgados & $35<40$ & $25<35$ & $15<25$ & $<15$ \\
\hline Total de pontos do reprodutor & $86<100$ & $66<86$ & $40<66$ & $<40$ \\
\hline
\end{tabular}

A avaliação do comportamento sexual consistiu no "teste 2" e foi realizada por meio do teste da libido, preconizado por Chenoweth (1984), adaptado para o touro Nelore por Pineda et al. (1997a). Os testes foram realizados em currais de tamanho médio $\left(400\right.$ a $\left.600 \mathrm{~m}^{2}\right)$, com duas vacas em estro e mais duas (ou três) vacas sem sinais de cio. Em seguida, introduzia-se um touro de cada vez no curral. O observador se mantinha atento ao comportamento do reprodutor e situado em posição estratégica, de modo que sua presença não fosse percebida pelo animal a ser avaliado.

A preparação das vacas para demonstração de estro consistiu de uma aplicação de $0,75 \mathrm{mg}$ $(3 \mathrm{~mL})$ de prostaglandina F2alpha (PGF2 $\alpha$; cloprostenol sódico), associada de uma aplicação de $10 \mathrm{mg}(5 \mathrm{~mL})$ de cipionato de estradiol (ECP), ambas por via intramuscular. Esse tratamento foi realizado 24 horas antes de se iniciarem as avaliações de comportamento sexual dos touros.
As aplicações foram realizadas em metade dos animais de manhã e o restante à tarde, de forma a ter sempre um número suficiente de vacas em estro durante todo o período de exame. O tempo de duração do teste de libido foi de 10 minutos. Entretanto, os touros que realizavam "uma monta completa" antes desse período foram liberados e recebiam a pontuação máxima. Os demais permaneciam em teste até vencerem o prazo e, então, recebiam notas de 0 a 10 , tendo-se em vista o seu desempenho, conforme Tab. 2.

Pela associação entre os resultados obtidos pelos parâmetros andrológicos (teste 1; Tab. 1) e exame de comportamento sexual (teste 2; Tab. 2), foi possível determinar o PR. Com base nesses resultados, foi proposto o número de vacas a lhes atribuir em estação de monta, variando de 60 a 120 dias de duração (Fonseca et al., 1991, 1997b, 2000; Franco et al., 2006), conforme Tab. 3 . 
Tabela 2. Pontuação para avaliação de comportamento sexual de touros Nelore (Bos indicus)

\begin{tabular}{|c|c|c|}
\hline Classe & Nota & Conduta sexual \\
\hline \multirow{3}{*}{ Questionável } & 0 & O touro não mostrou interesse sexual pela vaca ou vacas. Apático. \\
\hline & 1 & Identificou a vaca em cio (e.g., cheirou a vulva). \\
\hline & 2 & $\begin{array}{l}\text { Cheirou a vulva da vaca e a perseguiu insistentemente (e.g., comunicações olfato- } \\
\text { gustativas, seguidas de reflexo de Flehmen em diversas ocasiões). }\end{array}$ \\
\hline \multirow{4}{*}{ Bom } & 3 & $\begin{array}{l}\text { Intenção de monta sem salto, com mugido, deslocamento ou masturbação (e.g., } \\
\text { rodopio e/ou apoio do queixo sobre o dorso da vaca, com ou sem ligeira elevação } \\
\text { das patas dianteiras, contudo sem alcançá-la). }\end{array}$ \\
\hline & 4 & $\begin{array}{l}\text { Tentativa de monta sem pênis exposto (e.g., o touro salta sobre a vaca, porém não } \\
\text { consegue agarrá-la). }\end{array}$ \\
\hline & 5 & Uma tentativa de monta com pênis exposto. \\
\hline & 6 & Duas ou mais tentativas de monta com pênis exposto. \\
\hline \multirow[b]{2}{*}{ Muito bom } & 7 & Uma ou mais montas incompletas sem pênis exposto. \\
\hline & 8 & $\begin{array}{l}\text { Uma ou mais montas incompletas com pênis exposto (nesse caso, pode ocorrer } \\
\text { introdução ou não do pênis, mas não ocorre o arranque final que caracteriza a } \\
\text { cópula). }\end{array}$ \\
\hline \multirow{2}{*}{ Excelente } & 9 & $\begin{array}{l}\text { Uma monta completa (nesse caso, há introdução do pênis seguida do arranque } \\
\text { final). }\end{array}$ \\
\hline & 10 & $\begin{array}{l}\text { Duas montas completas, seguidas ou não por interesse sexual, incluindo tentativas } \\
\text { de monta, montas incompletas e novos serviços. }\end{array}$ \\
\hline
\end{tabular}

Tabela 3. Proposição para relação touro:vaca (número de vacas recomendado por touro), tendo-se como indicadores o teste andrológico propriamente dito (teste 1; Tab. 1) e a avaliação de comportamento sexual (teste 2; Tab. 2) de touros Nelore (Bos indicus)

\begin{tabular}{lllll}
\multicolumn{1}{c}{ Teste 2} & Excelente & Muito bom & Bom & Questionável \\
Teste 1 & & & & 50 \\
\hline Excelente & 80 a 100 & 70 & 50 & 40 \\
Muito bom & 60 & 55 & 40 & 30 \\
Bom & 50 & 05 & 0 & 0 \\
Questionável & 0 & 0 & \\
\hline
\end{tabular}

Fonte: adaptada de Fonseca (2000).

O teste para avaliação da independência dos fatores idade e comportamento sexual foi o de qui-quadrado de Pearson. O teste resultou no valor de $\chi 2=381,16$, com 12 graus de liberdade residuais e valor $\mathrm{P}<0,001$. Dessa forma, pode-se concluir que os fatores não são independentes. Para análise do efeito da classificação andrológica (i.e., aptos, inaptos temporários ou descarte) sobre as variáveis PE, Mot, V, Tb, DM, dm e TDE, os valores obtidos foram submetidos à análise de variância. Avaliou-se também o efeito da idade e sua interação com a classificação andrológica sobre o perímetro escrotal. O teste de Tukey-Kramer foi utilizado para as comparações de média, elencando-se erro tipo 1 igual a $5 \%$ de probabilidade. As análises estatísticas e os gráficos foram desenvolvidos em linguagem R (R Development..., 2017).

\section{RESULTADOS E DISCUSSÃO}

O aumento do parênquima testicular ocorre com o decorrer da maturidade sexual (Ayala et al., 2016; Fonseca et al., 2019), processo associado com desenvolvimento testicular, multiplicação de células germinativas (Brito et al., 2004), proliferação de células de Leydig e aumento dos níveis de testosterona (Araguren-Méndez et al., 1995; Dias et al., 2014; Ayala et al., 2016). Há décadas se reconhece que o PE apresenta alta correlação com volume e peso testiculares (Willet e Ohms, 1975; Pastore et al., 2008; Siqueira et al., 2012). Além do aumento do PE, a cor do pelame também se intensifica com a idade (Fonseca et al., 2019), características influenciadas pelos níveis de testosterona que se elevam à medida que a idade avança (Devkota $e t$ al., 2008). 
De acordo com o exame andrológico, o número de touros classificados como aptos consistiu em $88,9 \%$ dos animais avaliados $(\mathrm{n}=5480)$, dos quais $51,6 \%$ foram considerados excelentes $(n=2827)$, $41,2 \%$ muito bons $(n=2257)$ e $7,2 \%$ bons $(\mathrm{n}=394)$. Entre os animais questionáveis $(\mathrm{n}=682$; $11,1 \%), 79,6 \%$ foram classificados como inaptos temporários $(n=542)$ e $20,4 \% \quad(n=139)$ foram animais descarte. A avaliação andrológica utilizada neste estudo foi definida de acordo com a classificação reprodutiva apresentada na Tab. 1 (Fonseca et al., 1997a), a qual foi desenvolvida com base em tabelas classificatórias anteriores (Fonseca et al., 1989; Pineda et al., 1997a) e referenciada por vários autores (Vale Filho, 2001; Fonseca, 2000, 2009; Franco et al., 2006). Os resultados encontram-se demonstrados nas Tab. 4 e 5 .

Tabela 4. Valores médios e desvio-padrão do perímetro escrotal (PE), motilidade (Mt), vigor (V), turbilhonamento $(\mathrm{Tb})$, defeitos maiores $(\mathrm{DM})$, defeitos menores $(\mathrm{dm})$ e do total de defeitos espermáticos (TDE), de acordo com a classificação andrológica (A=aptos; Q1=inaptos temporários; Q2=descarte), independentemente da idade, de touros Nelore (Bos indicus) criados a pasto

\begin{tabular}{llll}
\hline & \multicolumn{2}{c}{ Classificação andrológica } & Q2 \\
\hline $\mathrm{n}$ & $\mathrm{A}$ & $\mathrm{Q} 1$ & 106 \\
$\mathrm{PE}(\mathrm{cm})$ & 5480 & 576 & $27,72 \pm 3,27^{\mathrm{c}}$ \\
$\mathrm{Mt}(\%)$ & $36,47 \pm 2,71^{\mathrm{a}}$ & $34,90 \pm 3,76^{\mathrm{b}}$ & $64,81 \pm 19,70^{\mathrm{b}}$ \\
$\mathrm{V}$ & $73,50 \pm 9,03^{\mathrm{a}}$ & $47,31 \pm 24,53^{\mathrm{c}}$ & $3,36 \pm 1,48^{\mathrm{b}}$ \\
$\mathrm{Tb}$ & $4,20 \pm 0,56^{\mathrm{a}}$ & $3,10 \pm 1,09^{\mathrm{c}}$ & $0,82 \pm 1,20^{\mathrm{b}}$ \\
$\mathrm{DM}(\%)$ & $1,99 \pm 1,51^{\mathrm{a}}$ & $0,55 \pm 1,26^{\mathrm{b}}$ & $14,06 \pm 14,91^{\mathrm{b}}$ \\
$\mathrm{dm}(\%)$ & $3,01 \pm 2,56^{\mathrm{c}}$ & $18,83 \pm 17,31^{\mathrm{a}}$ & $8,54 \pm 8,27^{\mathrm{b}}$ \\
$\mathrm{TDE}(\%)$ & $4,63 \pm 4,21^{\mathrm{c}}$ & $20,14 \pm 13,94^{\mathrm{a}}$ & $22,59 \pm 17,95^{\mathrm{b}}$ \\
\hline
\end{tabular}

Letras distintas na mesma linha indicam $\mathrm{P}<0,05$.

Com relação aos aspectos físicos do sêmen, os animais que apresentaram classificação A na presente avaliação (ou seja, touros aptos; Mot=73,5\% e V=4,2; Tab. 4) revelaram-se superiores aos touros A (aptos) avaliados por Fonseca et al. (1997a) (Mot=63,0\% e V=3,8). Essa constatação demonstra a evolução da qualidade espermática dos touros dessa raça, favorecida pela seleção baseada em tabelas classificatórias. Para touros classificados como A nesta pesquisa, os valores de motilidade variaram de $50 \%$ a $85 \%$ e de vigor entre 3 e 5 pontos, semelhantemente à sugestão de Vale Filho et al. (2012) para esses parâmetros.

Para morfologia espermática, na faixa etária de 12 a 18 meses, os resultados observados para defeitos maiores (DM) e total de defeitos espermáticos (TDE) demonstrados por Fonseca et al. (2019) ( $\mathrm{DM}=9,2 \%$ e $\mathrm{TDE}=16,2 \%)$, foram inferiores aos de Fonseca et al. (1997a) $(\mathrm{DM}=43,2 \%$ e $\mathrm{TDE}=57,4 \%)$. Esses resultados indicam progresso da precocidade reprodutiva no decorrer dos anos. De acordo com os critérios utilizados neste trabalho, um valor superior a $30 \%$ do total de alterações morfológicas sugere subfertilidade, o que difere dos critérios adotados por Vale Filho et al. (2012), que consideram regulares até $59 \%$ de defeitos espermáticos para touros nessa faixa etária.

$\mathrm{Na}$ Tab. 4, fica também evidente que maiores PE são observados em touros classificados como aptos. Da mesma forma, a motilidade e o vigor são superiores, e os defeitos espermáticos inferiores em animais dessa categoria. Por outro lado, animais com potencial reprodutivo questionável (inaptos temporários ou descarte) apresentam valores inferiores para essas características, o que comprova a importância dessa classificação para a eficiência reprodutiva de animais de produção.

Quanto ao PE dos animais aprovados (aptos), independentemente da idade (Tab. 4), evidente diferença foi observada entre os dados aqui demonstrados e aqueles anteriormente apresentados por outros trabalhos de pesquisa (Fonseca et al., 1997a; Fonseca, 2000; Franco et al., 2006). Os animais considerados aptos nas décadas de 90/2000 apresentavam PE médio de $33,0 \mathrm{~cm}$, ao passo que os aqui apresentados demonstram média de $36,5 \mathrm{~cm}$. Essa constatação evidencia a eficiência do exame andrológico na seleção de animais melhoradores. Adicionalmente, confirmam-se a alta repetibilidade e herdabilidade dessa característica 
(Fonseca, 2009) e a importância de se aplicar tabelas classificatórias atualizadas. Para os animais classificados como Q1, ou seja, animais portadores de alterações espermáticas ou testiculares adquiridas, não houve diferença entre os dados de PE aqui analisados e aqueles obtidos em $1997(34,90 \mathrm{~cm} \times 35,12 \mathrm{~cm})$ (Fonseca et al., 1997a).

A alta repetibilidade dos parâmetros Mot., V, DM e TDE (Fonseca, 2009) demonstra que a decisão de descartar touros Q2 (portadores de alterações consideradas insanáveis e/ou de origem genética), assim como aqueles com reduzido $\mathrm{PE}$, tem sido correta, revelando cada vez menor percentagem de touros com baixo potencial para fertilidade. $\mathrm{Na}$ Tab. 5, é possível observar que a média do PE de animais adultos (>48 meses), quando considerados descarte, apresentou valor semelhante ao de animais púberes aprovados (12 a 24 meses de idade).

Tabela 5. Valores médios e desvio-padrão do perímetro escrotal (PE) de animais separados por idade (meses), de acordo com as diferentes classificações andrológicas (A=aptos; Q1=inaptos temporários; $\mathrm{Q} 2=$ descarte) de touros Nelore (Bos indicus) criados a pasto

\begin{tabular}{|c|c|c|c|c|c|c|c|c|}
\hline & & \multicolumn{7}{|c|}{ Classificação andrológica } \\
\hline & & $\mathrm{A}$ & & Q1 & & Q2 & Total & \\
\hline Idade & $\mathrm{n}$ & $\mathrm{PE}(\mathrm{cm})$ & $\mathrm{n}$ & $\mathrm{PE}(\mathrm{cm})$ & $\mathrm{N}$ & $\mathrm{PE}(\mathrm{cm})$ & $\mathrm{n}$ & $(\%)$ \\
\hline $18<24$ & 668 & $32,56 \pm 2,54^{\mathrm{a}}$ & 118 & $30,82 \pm 3,89^{a}$ & 33 & $26,70 \pm 2,87^{\mathrm{a}}$ & 819 & 13,29 \\
\hline $24<36$ & 1067 & $33,96 \pm 2,84^{b}$ & 126 & $32,42 \pm 4,23^{\mathrm{b}}$ & 37 & $26,66 \pm 2,30^{\mathrm{a}}$ & 1230 & 19,96 \\
\hline $36<48$ & 647 & $36,83 \pm 2,91^{\mathrm{c}}$ & 69 & $36,40 \pm 3,61^{\mathrm{cd}}$ & 9 & $30,00 \pm 3,53^{\mathrm{ab}}$ & 725 & 11,76 \\
\hline Total & 5480 & & 576 & & 106 & & 6162 & 100,00 \\
\hline
\end{tabular}

Letras distintas na coluna denotam efeito significativo da idade sobre o perímetro escrotal dentro de cada classe andrológica $(\mathrm{P}<0,05)$.

A Tab. 5 evidencia que a idade do touro teve influência no PE. Os resultados desta pesquisa mostram que as médias de PE aqui apresentadas foram superiores às verificadas por Fonseca et al. (1989) para touros classificados como excelentes, bem como aos dados apresentados por Fonseca et al. (1997a), Fonseca (2000) e Vale Filho et al. (2012). Tais resultados demonstram a importância das diferentes tabelas de classificação que evoluem a seu tempo e informam a necessidade de aumentar a exigência dos parâmetros andrológicos de touros em estudo.

De acordo com Siqueira et al. (2012), a forma testicular alongada foi a prevalente em animais Nelore com idade média de 21 meses. Para o prognóstico do tamanho testicular em animais Nelore, a seleção por circunferência (ou perímetro) escrotal é medida adequada e apresenta adequada confiabilidade na predição do tamanho testicular, mesmo com a maior frequência de animais com testículos longos. Pastore et al. (2015), usando o recurso da ultrassonografia, não encontraram testículos "acentuadamente alongados" (0\%). A predominância foi do formato "moderado oval" $(61,4 \%)$, seguido do "longo moderado" (32,2\%).
O formato "oval esférico" atingiu 6,1\%, e, finalmente, o esférico $0,3 \%$, o que confirma a utilização do PE para estimar tamanho testicular (Willet e Ohms, 1975), dispensando utilização de medidas menos práticas e mais sujeitas a erros, como comprimento e largura.

Outro dado interessante está relacionado aos parâmetros de comportamento sexual (Tab. 2) dos touros da raça Nelore do presente estudo, quando os animais foram separados de acordo com sua classificação comportamental (Tab. 6 e Fig. 1).

O teste da libido, também denominado exame do comportamento sexual, demonstrou influência significativa e positiva da idade do touro. Ao se observar a Tab. 6 e a Fig. 1, nota-se que a proporção de animais em cada categoria foi alterada em função da sua evolução para a maturidade. Nesse sentido, o teste estatístico para avaliação da independência dos fatores idade e comportamento sexual demonstrou que os fatores não são independentes, confirmando as variações individuais entre touros da raça Nelore de diferentes idades, caráter genético e manejo a campo. 


\section{Potencial reprodutivo}

Tabela 6. Tabela de contingência por idade e categoria de comportamento sexual de touros Nelore (Bos indicus) criados a pasto

\begin{tabular}{lllllll}
\hline \multicolumn{7}{c}{ Faixas etárias (meses) } \\
$\begin{array}{llllll}\text { Comportamento } \\
\text { sexual }\end{array}$ & $12-18$ & $18-24$ & $24-36$ & $36-48$ & $>48$ & $\begin{array}{l}\text { Total } \\
\text { (por categoria) }\end{array}$ \\
Questionável & $\mathrm{n}=8$ & $\mathrm{n}=327$ & $\mathrm{n}=360$ & $\mathrm{n}=136$ & $\mathrm{n}=524$ & 1355 \\
& $(0,59 / 27,59)$ & $(24,13 / 40,02)$ & $(26,57 / 29,9)$ & $(10,04 / 19,1)$ & $(38,67 / 15,84)$ & 135 \\
Bom & $\mathrm{n}=11$ & $\mathrm{n}=276$ & $\mathrm{n}=395$ & $\mathrm{n}=270$ & $\mathrm{n}=1195$ & 2147 \\
& $(0,51 / 37,93)$ & $(12,86 / 33,78)$ & $(18,4 / 32,81)$ & $(12,58 / 37,92)$ & $(55,66 / 36,12)$ & \\
Muito bom & $\mathrm{n}=9$ & $\mathrm{n}=143$ & $\mathrm{n}=279$ & $\mathrm{n}=149$ & $\mathrm{n}=672$ & 1252 \\
& $(0,72 / 31,03)$ & $(11,42 / 17,5)$ & $(22,28 / 23,17)$ & $(11,9 / 20,93)$ & $(53,67 / 20,31)$ & \\
Excelente & $\mathrm{n}=1$ & $\mathrm{n}=71$ & $\mathrm{n}=170$ & $\mathrm{n}=157$ & $\mathrm{n}=917$ & 1316 \\
\hline Total (por idade) & $(0,08 / 3,45)$ & $(5,4 / 8,69)$ & $(12,92 / 14,12)$ & $(11,93 / 22,05)$ & $(69,68 / 27,72)$ & 6070 \\
\hline
\end{tabular}

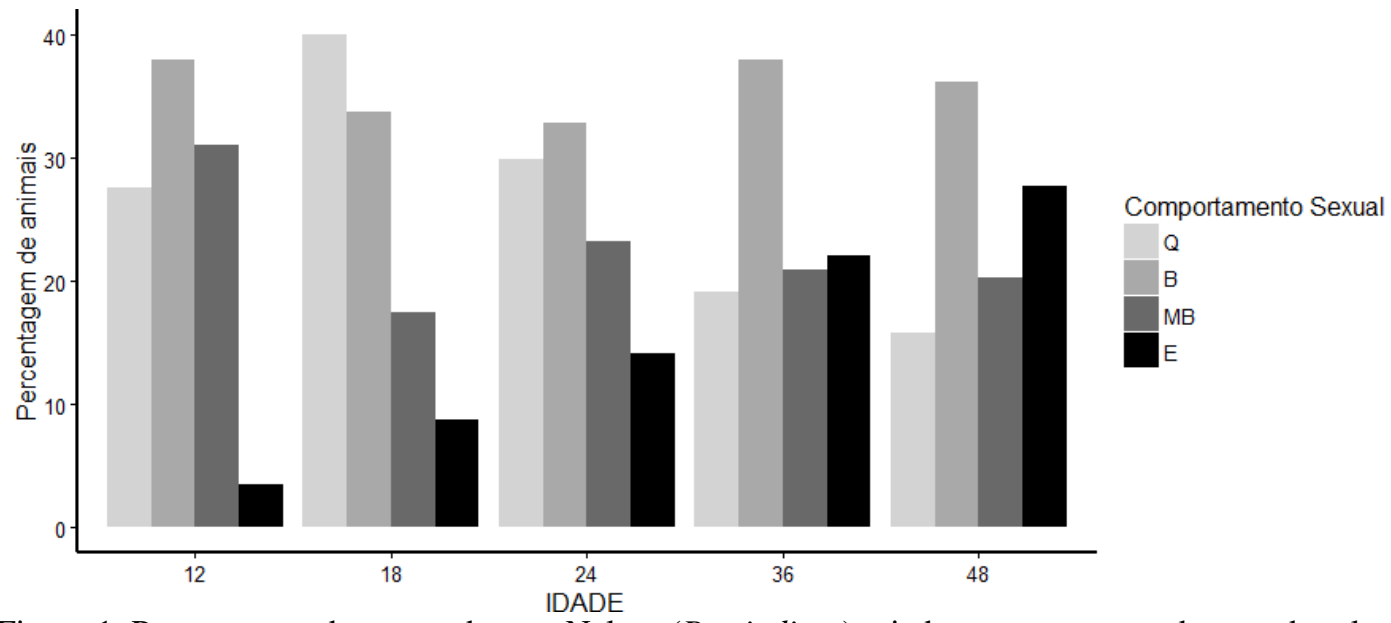

Figura 1. Percentagem de touros da raça Nelore (Bos indicus) criados a pasto, em cada uma das classes de comportamento sexual $(\mathrm{Q}=$ questionável, $\mathrm{B}=$ =bom, $\mathrm{MB}=$ muito bom, $\mathrm{E}=$ excelente), de acordo com a idade (em meses).

O primeiro valor fora dos parênteses em cada célula corresponde à contagem de animais; o primeiro valor dentro dos parênteses corresponde à percentagem de animais de cada idade dentro de cada categoria, enquanto o segundo corresponde à percentagem de animais de cada categoria dentro de cada idade. Os dados utilizados na determinação do PR dos touros aqui analisados tiveram por base o seu rendimento no exame andrológico, associado ao comportamental (variando entre questionável e excelente), na busca de se verificarem as variações deles nessa associação (Tab. 3). Os resultados estão apresentados na Tab. 7.

Tabela 7. Potencial reprodutivo (PR) de touros Nelore (Bos indicus) criados a pasto, avaliados em fazendas de diferentes estados brasileiros

\begin{tabular}{lllll}
\hline PR (vacas/touro) & n touros & $\%$ de animais & Classificação & Total (\%) \\
\hline 0 & 687 & 11,15 & Questionável & 11,15 \\
\hline 30 & 160 & 2,60 & & \\
40 & 674 & 10,94 & Bom & 35,84 \\
45 & 86 & 1,40 & & \\
50 & 1288 & 20,90 & & \\
\hline 55 & 472 & 7,66 & Muito bom & 40,81 \\
60 & 1450 & 23,54 & & 12,20 \\
70 & 593 & 9,61 & Excelente & 100 \\
\hline 80 a 100 & 752 & 12,20 & & \\
\hline Total & 6162 & 100 & & \\
\hline
\end{tabular}

Para classificação do PR, foram considerados o teste 1 (andrológico) e o teste 2 (comportamental). 
A presente avaliação do PR (Tab. 7), no que se refere ao teste da libido, foi realizada com base nos trabalhos de Chenoweth (1984) e, no Brasil, sofreu as adaptações para servir, também, a touros indianos (Pineda et al., 1997a). A classificação utilizada nesta pesquisa (Tab. 2) considera que dois reflexos de Flehmen (com ou sem perseguição da fêmea) são atitudes que não merecem mais do que a classificação de "questionáveis", com nota máxima de 2 pontos em 10 possíveis, o que difere do proposto por Vale-Filho et al. (2012).

Conforme descrito anteriormente, o número de touros classificados como excelentes, independentemente do teste da libido, foi 2827 (45,9\% do total de 6162 animais). Interessante observar que esse número foi reduzido para 752 $(12,2 \%)$ quando os dados de comportamento sexual foram incluídos para definição do seu PR. Nota-se que essa avaliação deve ser considerada mais um elemento fundamental na determinação do PR do reprodutor. Entretanto, julga-se importante salientar que o teste da libido, ao contrário do andrológico, não é desclassificatório. Todavia, funciona como importante ferramenta na determinação correta do PR, uma vez que a libido (Tab. 6) foi efetivamente influenciada pela idade do animal, aumentando à medida que o touro evoluía em sua maturidade sexual. Adicionalmente, destaca-se que o comportamento sexual foi bastante variável mesmo em animais maduros (Fig. 1). Visto que a libido apresenta alta correlação com a capacidade de serviço (Blockey, 1981), salienta-se a importância de considerá-la na composição do PR dos touros. Ainda, maior crescimento testicular se relaciona a maior número de células esteroidogênicas, maior quantidade de hormônios reprodutivos e, portanto, maior libido (Chenoweth, 1984).

A constatação de inúmeros resultados positivos, quando associados à alta repetibilidade do PE em touros Nelore $(r=0,66)$ (Fonseca, 2009) e suas correlações positivas com outros parâmetros andrológicos (Fonseca et al., 2019), ganhos genéticos aditivos (Crudeli et al., 1992; Fonseca et al., 1997b, 2000) e, possivelmente, comportamentais (Tab. 6 e 7), denota a importância da aplicação de tabelas classificatórias como método simples de seleção para touros Nelore. Percebe-se ainda sensível avanço genético da raça no que diz respeito ao seu PR. Portanto, o progresso das tabelas classificatórias foi ferramenta útil na seleção de touros criados a campo, uma vez que condições genéticas e ambientais influenciam o potencial reprodutivo do touro.

A classificação do PR estabelecido na Tab. 7 para a determinação do número de vacas que um touro pode servir em estação de monta (entre 60 e 120 dias de duração) foi demonstrada por diversos pesquisadores por meio de testes a campo, o que dá suporte a essa divulgação (Crudeli et al., 1992; Fonseca et al., 1997b, 2000; Pineda et al., 1997b; Fonseca, 2000; Salvador et al., 2002; Santos et al., 2004; Franco et al., 2006). O aumento da proporção vaca:touro em animais com alto PR, avaliados com a associação dos parâmetros andrológicos e do comportamento sexual (conforme aqui proposto), não acarretou queda da taxa de concepção (Fonseca et al., 1997a , 2000; Franco et al., 2006). Franco et al. (2006) compararam touros com alto $\mathrm{PR}$, o que os credenciava para atender até $70 \mathrm{e}$ 80 vacas durante a estação de monta. As taxas de concepção, obtidas pelos touros do grupo experimental $(1: 100)$ e pelos touros do grupo controle (1:50), não divergiram entre si $(87 \%$ e $88 \%$, respectivamente). Por fim, a nova tabela classificatória proposta neste artigo (Tab. 8) tem base nos parâmetros encontrados nesta pesquisa visando o progresso da raça Nelore.

Assim, salienta-se que o uso de tabela de classificação andrológica por pontos com parâmetros atualizados, de modo a exigir melhores índices em cada renovação, traz benefícios para a seleção de touros da raça Nelore. Ainda, a utilização do teste da libido é ferramenta importante para a correta determinação do PR de cada touro, a qual possibilita maiores ganhos genéticos aditivos e redução dos custos com o rebanho. Dessa forma, touros da raça Nelore, com esses cuidados e associados a práticas de seleção mais complexas, caminham no sentido de alcançar maiores índices de fertilidade. 
Tabela 8. Classificação indicada para touros Nelore (Bos indicus) com base nas médias e desvios-padrão dos parâmetros andrológicos de reprodutores em uso no campo ( $\mathrm{n}=6162$ animais) de diferentes fazendas brasileiras. Uma nova proposição

\begin{tabular}{|c|c|c|c|c|}
\hline \multirow{2}{*}{ Parâmetros } & \multicolumn{4}{|c|}{ Classificação } \\
\hline & Excelente & Muito bom & Bom & Questionável \\
\hline \multicolumn{5}{|l|}{ 1-Motilidade espermática } \\
\hline Motilidade progressiva (\%) & $\geq 75$ & $60<75$ & $50<60$ & $<50$ \\
\hline Vigor & 5 & $4<5$ & $3<4$ & $<3$ \\
\hline Turbilhonamento & $4 \leq 5$ & $3<4$ & $2<3$ & $<2$ \\
\hline Pontos outorgados & $21 \leq 25$ & $16<21$ & $10<16$ & $<10$ \\
\hline Defeitos maiores $(\%)$ & $\leq 3$ & $4 \leq 10$ & $11 \leq 15$ & $>15$ \\
\hline Defeitos totais $(\%)$ & $\leq 10$ & $11 \leq 15$ & $16 \leq 30$ & $>30$ \\
\hline Pontos outorgados & $30 \leq 35$ & $25<30$ & $20<25$ & $<20$ \\
\hline \multicolumn{5}{|l|}{ 3-Perímetro escrotal (cm) } \\
\hline $12<18$ meses & $\geq 33,5$ & $31,5<33,5$ & $29,5<31,5$ & $<29,5$ \\
\hline $18<24$ meses & $\geq 34,0$ & $32,0<34,0$ & $30,5<32,0$ & $<30,5$ \\
\hline Pontos outorgados & $35 \leq 40$ & $30<35$ & $15<30$ & 0 \\
\hline Total de pontos do reprodutor & $86 \leq 100$ & $71<86$ & $45<71$ & $<45$ \\
\hline
\end{tabular}

Para animais coletados com eletroejaculador, a nota de turbilhonamento não deve ser considerada para a pontuação do parâmetro 1 .

\section{CONCLUSÕES}

Tendo em vista o desempenho de touros Nelore nos testes andrológico e de comportamento sexual associados para avaliação do potencial reprodutivo (PR) em testes de monta a campo do presente estudo, conclui-se que a ausência do exame de libido (comportamento sexual) não permite a definição correta do PR e acaba por reforçar e/ou conservar a tradição do criador de utilizar baixas proporções de vacas por reprodutor (1:30 ou menos). Adicionalmente, o trabalho confirma a importância da criteriosa avaliação do reprodutor, pois permite melhor utilização dos reprodutores e seleção mais apurada deles. Além da melhoria dos parâmetros andrológicos com o avançar da idade, a evolução dessas características foi também observada pela seleção genética das últimas décadas.

\section{REFERÊNCIAS}

AHMAD, N.; UMAIR, S.; SHAHAB, M.; ARSLAN, M. Testicular development and establishment of spermatogenesis in Nili-Ravi buffalo bulls. Theriogenology, v.73, p.20-25, 2010.

ARAGUREN-MÉNDEZ, J.; MADRID-BURY, N.; GONZÁLEZ-STAGNARO, C. et al. Pubertad em toretes 5/8 Holstein y 5/8 Pardo suizo. Rev. Facul. Agron., v.12, p.393-407, 1995.
AYALA, H.D.M.; RIBEIRO, H.F.L.; ROLIM FILHO, S.T. et al. Association of testicular echogenicity, scrotal circumference, testicular volume and testosterone concentration in buffaloes. Rev. Bras. Med. Vet., v.38, p.334-340, 2016.

BERGMANN, J.A.G.; ZAMBORLINI, L.C.; PROCÓPIO, C.S.O. et al. Estimativas de parâmetros genéticos do perímetro escrotal e do peso corporal em animais da raça Nelore. Arq. Bras. Med. Vet. Zootec., v.48, p.69-78, 1996.

BLOCKEY, M.A.B., STRAW, W.M.; JONES, L.P. Heritability of serving capacity and scrotal circumference in beef bulls. In: 70th Annu. Mtg. Am. Soc. Anim. Sci., East Lansing, MI, p.92, 1978.

BLOCKEY, M.A.B. Development of a serving capacity test for beef bulls. Appl. Anim. Ethol. v.7, p.307-319, 1981.

BLOM, E. The ultrastructure of same charactheristics sperm defects and a proposal of a new classification of bull spermiogram. Nord. Vet. Med., v.25, p.383-391, 1973.

BRITO, L.F.C.; SILVA, A.E.D.F.; UNANIAN, M.M. et al. Sexual development in early- and late-maturing Bos indicus and Bos indicus x Bos taurus crossbred bulls in Brazil. Theriogenology, v.62, p.1198-1217, 2004. 
CHENOWETH, P.J. Examination of bulls for libido and breeding ability. Vet. Clin. N. Am. Large Anim. Pract., v.5, p.59-74, 1984.

CRUDELI, A.G.; FONSECA, V.O.; COSTA e SILVA, E.V.; HERMANY, A. Aptidão reprodutiva de touros da raça Nelore. Efeito das características seminais e circunferência escrotal sobre a fertilidade. Rev. Bras. Reprod. Anim., v.16, p.33-39, 1992.

DEVKOTA, B.; KOSEKI, T.; MATSUI, M. et al. Relationships among age, body weight, scrotal circumference, semen quality and peripheral testosterone and estradiol concentrations in pubertal and postbubertal Holstein bulls. J. Vet. Med. Sci., v.70, p.119-121, 2008.

DIAS, J.C.; EMERICK, L.L.; ANDRADE, V.J. et al. Concentrações séricas de testosterona em touros jovens Guzerá e suas associações com características reprodutivas. Arch. Vet. Sci., v.19, p.24-31, 2014.

FONSECA, V.O. Avaliação reprodutiva de touros para monta a campo: análise crítica. Rev. Bras. Reprod. Anim., v.33, Supl.6, p.36-41, 2009.

FONSECA, V.O. O touro no contexto da eficiência reprodutiva do rebanho. Inf. Agropecu., v.21, p.48-63, 2000.

FONSECA, V.O.; BERGMANN, J.A.G.; FRANCO, C.S. Potencial reprodutivo de touros Nelore acasalados com elevado número de vacas. Arq. Bras. Med. Vet. Zootec., v.49, p.53-62, 1997b.

FONSECA, V.O.; COSTA e SILVA, E.V.; HERMANNY, A. Classificação andrológica de touros zebus com base na biometria testicular e características morfo-físicas do sêmen. Rev. Bras. Reprod. Anim., v.13, Supl.1, p.187, 1989.

FONSECA, V.O.; CRUDELI, G.A.; COSTA e SILVA, E.V. Potencial reprodutivo de touros da raça Nelore (Bos taurus indicus) em monta natural: proporção touro:vaca 1:40 e fertilidade. Rev. Bras. Reprod. Anim., v.15, p.103-108, 1991.

FONSECA, V.O; FRANCO, C.S.; BERGMANN, J.A.G. Potencial reprodutivo e econômico de touros Nelore acasalados coletivamente na proporção de um touro para 80 vacas. Arq. Bras. Med. Vet. Zootec., v.52, p.77$82,2000$.
FONSECA, V.O.; SANTOS, N.R.; MALINSKI, P.R. Classificação andrológica de touros zebus com base no perímetro escrotal e características morfo-físicas do sêmen. Rev. Bras. Reprod. Anim., v.21, p.36-39, 1997a.

FONSECA, V.O.; SOUZA, C.F.; AZEVEDO, N.A. et al. Parâmetros reprodutivos de touros Nelore (Bos taurus indicus) criados a pasto de diferentes faixas etárias. Arq. Bras. Med. Vet. Zootec., v.71, p.385-392, 2019.

FRANCO, C.S.; FONSECA, V.O.; GASTE, L. Potencial reprodutivo de touros Nelore acasalados coletivamente na proporção de um touro para 100 vacas. Arq. Bras. Med. Vet. Zootec., v.58, p.1156-1161, 2006.

MANUAL para exame andrológico e avaliação de sêmen animal. 2.ed. Belo Horizonte: CBRA, 1998. 49p.

PASTORE, A.A.; TONIOLLO, G.H.; LÔBO, R.B. et al. Características biométricas, testiculares, seminais e parâmetros genéticos de touros pertencentes ao programa de melhoramento genético da raça Nelore. ARS Vet., v.24, p.134-141, 2008.

PASTORE, A.A.; TONIOLLO, G.H.; CARDILLI, D.J. et al. Contribuição da ultrassonografia na avaliação andrológica de bovinos Nelore. Rev. Bras. Reprod. Anim., v.39, p.32-40, 2015.

PEREIRA, E.; ELER, J.P.; FERRAZ, J.B.S. Análise genética de características reprodutivas na raça Nelore. Pesqui. Agropecu. Bras., v.37, p.703-708, 2002.

PEREIRA, J.C.C. Melhoramento genético aplicado à produção animal. 6.ed. Belo Horizonte: FEPMVZ, 2012. [758p.].

PINEDA, N.R.; FONSECA, V.O.; PROENÇA, R.V. Potencial reprodutivo de touros de alta libido da raça Nelore (Bos taurus indicus). Rev. Bras. Reprod. Anim., v.22, p.45-48, 1997 b.

PINEDA, N.R.; LEMOS, P.F.; FONSECA V.O. Comparação entre dois testes de avaliação comportamental sexual (libido) de touros Nelore (Bos taurus indicus). Rev. Bras. Reprod. Anim., v.21, p.29-34, 1997a.

R DEVELOPMENT core team: a language and environment for statistical computing. Vienna: R Foundation for Statistical Computing, 2017. 
SALVADOR, D.F.; DIAS, J.C.; VALE-FILHO V.R. et al. Perfil Andrológico de touros da raça Nelore com três e quatro ano de idade, criados extensivamente em condições do estado do Mato Grosso do Sul. Rev. Bras. Reprod. Anim., v.26, p.64-65, 2002.

SANTOS, M.D.; TORRES, C.A.A.; RUAS, J.R.M. et al. Potencial reprodutivo de touros da raça Nelore submetidos a diferentes proporções touro: vaca. Arq. Bras. Med. Vet. Zootec., v.56, p.497-503, 2004.

SIQUEIRA, J.B.; OBA, E.; PINHO, R.O. et al. Testicular shape and andrological aspects of young Nellore bulls under extensive farming. Rev. Bras. Zootec., v.41, p.612-617, 2012.

VALE-FILHO, V.R. Subfertilidade em touros jovens e erados: parâmetros para avaliação andrológica e conceituação geral. Cad. Tecn. Vet. Zootec., v.35, p.81-87, 2001.
VALE-FILHO, V.R.; ANDRADE， V.J.; AZEVEDO, N.A. Avaliação andrológica e seleção de tourinhos zebu para reprodução. In: SIMPÓSIO INTERNACIONAL DE PRODUÇÃO DE GADO DE CORTE (SIMCORTE). 7., 2010, Viçosa. Anais... Viçosa: UFV / DZO, 2010. p.363-412.

VALE-FILHO, V.R.; ANDRADE, V.J.; SILVA, M.A. et al. Novos métodos de avaliação andrológica e do potencial reprodutivo de touros. In: SIMPÓSIO NACIONAL SOBRE PRODUÇÃO E GERENCIAMENTO DA PECUÁRIA DE CORTE. 5., 2012, Belo Horizonte. Anais... Belo Horizonte: UFMG, 2012. p.179-239.

WILLET, E.L.; OHMS, J.I. Measurement of testicular size and its relations to reproduction of spermatozoa by bulls. J. Dairy Sci., v.40, p.15591569, 1975. 